\title{
Hepatitis $C$ virus core protein substitutions affect the response to pegylated-interferon and ribavirin therapy
}

\author{
Fatimah S. Alhamlan ${ }^{1}$, Mohammed N. Al-Ahdal ${ }^{1,2,3}$, Nisreen Z. Khalaf ${ }^{1}$, Ayman A. Abdo ${ }^{4,5}$, Faisal Sanai ${ }^{6}$, \\ Hamad I. Al-Ashgar ${ }^{7}$, Ahmed A. Al-Qahtani ${ }^{1,3,4^{*}}$ \\ From 2nd International Genomic Medical Conference (IGMC 2013) \\ Jeddah, Kingdom of Saudi Arabia. 24-27 November 2013
}

\section{Background}

Hepatitis $\mathrm{C}$ virus (HCV) shows remarkable genetic diversity, which contributes to its high persistence and varied susceptibilities to antiviral treatments. Previous studies have reported that the substitution of amino acids in the $\mathrm{HCV}-1 \mathrm{~b}$ core region at positions 70 (Arg70 to Gln70) and/or 91 (Lue91 to Met91) is associated with a poor response to pegylated- interferon and ribavirin (PEG-IFN/ RBV) therapy $[1,2]$. Because the role of the core protein in HCV infections is unclear in Saudi populations, we aimed in this study to analyze the full-length core protein sequences from Saudi patients.

\section{Materials and methods}

A total of 300 samples were obtained from Saudi patients who went through PEG-IFN/RBV treatment. Samples were divided further to responder and non-responder groups. Direct sequencing was employed, followed by sequence analyses using advanced software.

\section{Results}

Our data showed that there was significant association between core protein mutations, particularly at position 70 , and treatment outcome in HCV1b and HCV-4d but not in HCV-1a and HCV-4a clinical samples. In addition, amino acid residue at position 91 was well-conserved among all clinical samples where Cys91 is the dominant amino acid residue. Furthermore, our data reported point mutations at different positions that were flagged as 'rare' mutations by negative BLOSUM scores.

\footnotetext{
* Correspondence: aqahtani@kfshrc.edu.sa

'Department of Infection and Immunity, King Faisal Specialist Hospital and Research Center, Riyadh, Saudi Arabia

Full list of author information is available at the end of the article
}

\section{Conclusions}

Amino acid substitution pattern differs substantially among HCV sub-genotypes. Such discrepancy needs further investigations. Our finding provides a new insight into $\mathrm{HCV}$ among effected Saudi population where the knowledge of $\mathrm{HCV}$ polymorphisms is lacking.

\section{Authors' details}

'Department of Infection and Immunity, King Faisal Specialist Hospital and Research Center, Riyadh, Saudi Arabia. ${ }^{2}$ Department of Pathology and Laboratory Medicine, King Faisal Specialist Hospital and Research Center, Riyadh, Saudi Arabia. ${ }^{3}$ Department of Microbiology and Immunology, College of Medicine, Alfaisal University, Riyadh, Saudi Arabia. ${ }^{4}$ Liver Disease Research Center, King Saud University, Riyadh, Saudi Arabia. ${ }^{5}$ Section of Gastroenterology, Department of Medicine, College of Medicine, King Saud University, Riyadh, Saudi Arabia. ${ }^{6}$ Hepatobiliary Sciences and Liver Transplantation, King Abdulaziz Medical City, Riyadh, Saudi Arabia. ${ }^{7}$ Department of Medicine, King Faisal Specialist Hospital \& Research Center, Riyadh, Saudi Arabia.

Published: 2 April 2014

\section{References}

1. Akuta N, Suzuki F, Kawamura Y, Yatsuji H, Sezaki H, Suzuki Y, Hosaka T, Kobayashi M, Arase Y, Ikeda K, Kumada H: Predictive factors of early and sustained responses to peginterferon plus ribavirin combination therapy in Japanese patients infected with hepatitis $C$ virus genotype $1 \mathrm{~b}$ : amino acid substitutions in the core region and low-density lipoprotein cholesterol levels. J Hepatol 2007, 46:403-410.

2. El-Shamy A, Kim SR, Ide YH, Sasase N, Imoto S, Deng L, Shoji I, Hotta H: Polymorphisms of hepatitis $C$ virus non-structural protein $5 \mathrm{~A}$ and core protein and clinical outcome of pegylated-interferon/ribavirin combination therapy. Intervirology 2011, 55:1-11.

doi:10.1186/1471-2164-15-S2-P5

Cite this article as: Alhamlan et al: Hepatitis $C$ virus core protein substitutions affect the response to pegylated-interferon and ribavirin therapy. BMC Genomics 2014 15(Suppl 2):P5.

\section{()

